\title{
Peritonealdialyse - eine Alternative zur Hämodialyse im Zentrum
}

\author{
Andreas Vychytil · Walter H. Hörl
}

Online publiziert: 25. Juni 2013

(C) Springer-Verlag Wien 2013

Für Patienten mit terminaler Niereninsuffizienz stehen als Nierenersatzverfahren die Nierentransplantation, die Hämodialyse (HD) und die Peritonealdialyse (PD) zur Verfügung. Dieser Themenschwerpunkt ist der PD gewidmet. Zur Durchführung der PD wird ein dünner Kunststoffkatheter in die Bauchhöhle implantiert. Der PD-Katheter hat eine Muffe oder zwei Muffen, die in der Bauchwand fixiert wird/werden. Der externe Anteil des PD-Katheters tritt seitlich des Nabels (meist etwas oberhalb, seltener unterhalb der Gürtellinie) aus der Bauchwand heraus und wird unter der Kleidung getragen. Über den PD-Katheter wird einerseits Dialyselösung in die Bauchhöhle instilliert und andererseits die mit Urämietoxinen angereicherte Lösung auch wieder drainiert. Eine optimale Katheterimplantation ist von eminenter Wichtigkeit für die weiteren klinischen Ergebnisse, deshalb wird sie auch in einem eigenen Kapitel behandelt. Die Dialyselösung enthält verschiedene Elektrolyte, einen Puffer (Laktat, Bikarbonat oder eine Kombination aus beiden Puffern) sowie ein osmotisches Agens (Glukose, Aminosäuren oder Icodextrin). Das osmotische Agens ist notwendig, um einen osmotischen Gradienten zwischen peritonealen Kapillaren und Peritonealhöhle aufzubauen, der zu einem Flüssigkeitsentzug führt. Je nach Ultrafiltrationsbedarf werden Dialyselösungen mit drei verschiedenen Glukosekonzentrationen oder Icodextrin (Polyglukose) angeboten.

Univ. Prof. Dr. A. Vychytil $(\bowtie)$

Abteilung für Nephrologie und Dialyse,

Klinik für Innere Medizin III, Medizinische Universität Wien, Währinger Gürtel 18-20, 1090 Wien, Österreich

E-Mail: andreas.vychytil@meduniwien.ac.at

Univ. Prof. DDr. W. H. Hörl, FRCP

Abteilung für Nephrologie und Dialyse,

Klinik für Innere Medizin III, Medizinische Universität Wien,

Währinger Gürtel 18-20, 1090 Wien, Österreich

E-Mail: walter.hoerl@meduniwien.ac.at
Bei der kontinuierlichen ambulanten Peritonealdialyse (CAPD) führt der Patient nach entsprechender Einschulung zu Hause 3-4 mal täglich manuell einen Dialysatwechsel durch. Hierfür wird ein Doppelbeutelsystem an den PD-Katheter konnektiert. Zunächst erfolgt der Auslauf des an Urämietoxinen reichen Dialysates aus der Bauchhöhle in den Leerbeutel, dann über dasselbe Lumen (aber in umgekehrter Richtung) der Einlauf der frischen Dialyselösung in die Peritonealhöhle. Ein Dialysatwechsel dauert etwa 30 bis 45 min, der Zeitpunkt kann innerhalb gewisser Grenzen flexibel gestaltet werden. Die automatisierte Peritonealdialyse (APD) führt der Patient ebenfalls nach entsprechender Einschulung über Nacht zu Hause durch. Dabei wird aber ein Gerät (Cycler) zu Hilfe genommen, das nach Konnektion mit dem PD-Katheter durch den Patienten eine nächtliche Behandlung durchführt. Der Cycler führt während der APD mehrere Dialysezyklen durch, die jeweils aus einem Dialysateinlauf, der Dialysatverweilzeit in der Peritonealhöhle und dem Dialysatauslauf bestehen. In der Früh diskonnektiert sich der Patient wieder vom Gerät und ist dadurch tagsüber flexibel. Allerdings hängt die Tatsache, ob nun eine CAPD oder eine APD durchgeführt werden kann, auch von medizinischen Faktoren (z. B. von der Beschaffenheit und den Transporteigenschaften der Peritonealmembran) ab.

Seit den ersten akuten PD-Behandlungen in den 1920iger Jahren und der zunehmend größeren Verbreitung der chronischen PD in den 1970iger Jahren haben laufende Entwicklungen (z. B. im Bereich der Dialyselösungen) zu einer zunehmenden Verbesserung der klinischen Ergebnisse bei Behandlung mit diesem Verfahren geführt. Den Neuentwicklungen ist in diesem Band ein eigenes Kapitel gewidmet. Viele Daten zeigen, dass PD und HD vom Patientenüberleben her gleichwertige Dialyseverfahren sind. Klinische Studien berichten außerdem, dass ein relativ hoher Prozentsatz an dialysepflichtigen Patienten ein Heimdialyseverfahren bevorzugen würde, falls diese Behandlungsalternative angeboten würde. 
Auch die NephrologenInnen gaben in Befragungen an, dass sie die PD für eine ideale initiale Form der Nierenersatztherapie halten. Paradoxerweise wird aber in vielen Ländern nur ein geringer Prozentsatz der Patienten mit diesem Dialysevefahren behandelt, in Österreich etwa 8-9\% der Dialysepatienten. Die Ursache liegt vorwiegend in nicht-medizinischen, teils organisatorischen Barrieren. Ein wichtiger Punkt ist die oftmals zu späte Zuweisung zum Nephrologen. Adäquate Information der Patienten über die Nierenersatztherapie erfordert Zeit. Die Patienten müssen zunächst einmal ihre Dialysepflichtigkeit akzeptieren. Andernfalls ist der Beginn einer Heimtherapie, die ein eigenverantwortliches Arbeiten des Patienten erfordert, unmöglich und es kann dann nur die HD im Zentrum oder in der Dialysepraxis durchgeführt werden. Aber auch in weiterer Folge sind oft Informationsgespräche notwendig, um den Patienten bei der Wahl der Nierenersatztherapie zu unterstützen. Daher ist die rechtzeitige Zuweisung der Patienten mit chronischer Niereninsuffizienz zum Nephrologen von eminenter Bedeutung. Interessanterweise zeigen klinische Analysen, dass der Zeitpunkt der Zuweisung zum Nephrologen auch zwischen verschiedenen Fachrichtungen differiert. So haben beispielsweise Kardiologen oder Endokrinologen ihre Patienten später zugewiesen als Allgemeinmediziner. Der Bedeutung der Prädialyseinformation und Patienteninformation ist in diesem Band ebenfalls ein Kapitel gewidmet. Ein Patient, der akut dialysepflichtig ins Spital kommt, wird meistens über einen zentralen Venenkatheter hämodialysiert und wechselt in weiterer Folge nur selten das Verfahren. Dies hat vor allem zwei Gründe. Einerseits werden an den akut andialysierten Patienten oft keine weiteren Informationen über alternative Formen der Nierenersatztherapie mehr weitergegeben. Andererseits erlebt der Patient in diesem Fall auch oft die HD als jenes Verfahren, das ihm das Leben gerettet und zu einer deutlichen Besserung geführt hat und will dann nicht mehr wechseln. Auch der Hausarzt oder der Internist spielen bei der Verfahrenswahl eine wichtige Rolle. Nicht selten wird dem Patienten von einem Heimdialyseverfahren abgeraten. Möglicherweise basiert dies auf der mangelnden Information dieser Kollegen (ist doch im Vergleich zu kardiologischen Patienten der Dialysepatient in einer Allgemeinpraxis oder internistischen Praxis eher ein seltener Gast). Vielleicht besteht aber auch die Sorge, allzu sehr in die Betreuung dieser Patienten involviert zu werden, ohne die entsprechende Erfahrung zu haben. Hier wären eine enge Kooperation und ein Informationsaustausch zwischen Dialysezentren und niedergelassenen Kollegen von großer Bedeutung. Ein anderes Problem ist, dass vor allem kleinere Zentren zu restriktiv mit der Patientenwahl für die PD sind. Rezente Studien zeigen, dass aus medizinischer und psychologischer Sicht durchaus $70-80 \%$ der Patienten für dieses Verfahren geeignet wären. Klinische Daten berichten über gute klinische Ergebnisse bei Patientengruppen, die man früher eher aus dem PD-Programm ausgeschlossen hätte, z. B. Patienten mit Zystennieren oder jene mit chronischem Versagen des Nierentransplantates. Auch diesen Aspekten ist in diesem Band ein Kapitel gewidmet. Auf der anderen Seite gibt es Patientengruppen, die gut für die PD geeignet wären, diese aber nicht selbst durchführen können. Dazu zählen ältere Patienten und jene mit großer Co-Morbidität. Für beide Gruppen gilt, dass der Transport zur HD in das Zentrum drei mal pro Woche beschwerlich und in klinischen Studien bei längeren Wegstrecken sogar mit einer erhöhten Mortalität assoziiert ist. Wie in einem weiteren Kapitel dieses Bandes diskutiert, ermöglicht eine Assistenz durch Angehörige oder Pflegepersonen auch diesen Patienten die Durchführung einer PD zu Hause.

Neben all diesen Barrieren finden sich aber noch andere Gründe, warum die PD bei uns nur wenig Verbreitung findet. Zunächst ist die Refundierung der Behandlungskosten äußerst gering. Dem steht aber doch ein relativ großer Aufwand bei der Patientenbetreuung (z. B. die oft wochenlange Einschulung der Patienten) gegenüber. Daher ist der Beginn eines solchen Verfahrens für Krankenhäuser oft nicht attraktiv. Die Zahl der PD-Patienten korreliert auch invers mit der HD-Kapazität einer Dialyseabteilung (je mehr freie HD-Kapazität, desto weniger PD) und positiv mit der Gesamtzahl der in einem Zentrum behandelten Patienten (große Zentren akzeptieren eher, PD-Patienten zu betreuen). Schließlich spielt natürlich die nephrologische Ausbildung eine Rolle. In Zentren, die vorwiegend HD anbieten, ist die Erfahrung mit PD gering und Kollegen in Ausbildung müssen an andere Zentren geschickt werden, um letzteres Verfahren kennenzulernen. Nicht immer ist dies aufgrund der eingeschränkten Ressourcen möglich. Es entsteht dann eine Situation, in der es den Kollegen leichter fällt, den Patienten die HD näherzubringen, als ein Verfahren vorzustellen, dass sie nur wenig kennen und eventuell auch nur eingeschränkt anbieten können. Auch der Patient erlebt dann das Missverhältnis zwischen der Zahl der HD- und PD-Patienten und will natürlich den „Goldenen Standard" haben, und das ist aus seiner Sicht das Verfahren, das die meisten Patienten durchführen.

Zuletzt sollte noch erwähnt werden, dass PD und HD keine gegenseitig konkurrierenden Verfahren sein sollten. Es ist durchaus möglich, mit der PD zu beginnen und später (z. B. nach Rückgang der Nierenrestfunktion) an die HD zu wechseln. Dieses ,integrated care-Konzept" hat in klinischen Studien die Ergebnisse der dialysepflichtigen Patienten im Vergleich zu jenen, die nur HD durchführen, verbessert. Auch muss erwähnt werden, dass bei der Prädialyseinformation immer auch die Möglichkeit einer präemptiven Nierentransplantation in Erwägung gezogen werden sollte.

Dieser Themenschwerpunkt wendet sich bewusst auch an nicht-nephrologische Kollegen, wie z. B. Allgemeinmediziner oder Internisten. Wir hoffen, dass die Zusammenstellung der 5 Artikel, die wichtige Bereiche der PD-Therapie abdecken, für unsere Leser interessant ist und das Lesen entsprechend Freude bereitet.

\section{Interessenskonflikt}

Es besteht kein Interessenskonflikt seitens der Autoren. 